\title{
CASTIN: a system for comprehensive analysis of cancer-stromal interactome
}

\author{
Daisuke Komura', Takayuki Isagawa', Kazuki Kishi ${ }^{1,2}$, Ryohei Suzuki ${ }^{1,3}$, Reiko Sato ${ }^{1}$, Mariko Tanaka ${ }^{4}$, Hiroto Katoh ${ }^{1}$, \\ Shogo Yamamoto ${ }^{5}$, Kenji Tatsuno ${ }^{5}$, Masashi Fukayama ${ }^{4}$, Hiroyuki Aburatani ${ }^{5}$ and Shumpei Ishikawa ${ }^{1 *}$
}

\begin{abstract}
Background: Cancer microenvironment plays a vital role in cancer development and progression, and cancer-stromal interactions have been recognized as important targets for cancer therapy. However, identifying relevant and druggable cancer-stromal interactions is challenging due to the lack of quantitative methods to analyze whole cancer-stromal interactome.

Results: We present CASTIN (CAncer-STromal INteractome analysis), a novel framework for the evaluation of cancer-stromal interactome from RNA-Seq data using cancer xenograft models. For each ligand-receptor interaction which is derived from curated protein-protein interaction database, CASTIN summarizes gene expression profiles of cancer and stroma into three evaluation indices. These indices provide quantitative evaluation and comprehensive visualization of interactome, and thus enable to identify critical cancer-microenvironment interactions, which would be potential drug targets.

We applied CASTIN to the dataset of pancreas ductal adenocarcinoma, and successfully characterized the individual cancer in terms of cancer-stromal relationships, and identified both well-known and less-characterized druggable interactions.
\end{abstract}

Conclusions: CASTIN provides comprehensive view of cancer-stromal interactome and is useful to identify critical interactions which may serve as potential drug targets in cancer-microenvironment. CASTIN is available at: http://github.com/tmd-gpat/CASTIN.

Keywords: Cancer microenvironment, Cancer-stromal interactions, Xenograft mouse model, RNA-Seq

\section{Background}

Cancer cells generally survive in microenvironment surrounded by non-cancer "stromal" cells such as endothelial cells, fibroblasts and immune cells. Stromal cells in cancer microenvironment promote maintenance, growth and progression of cancer cells through the release of humoral factors and direct cell contact. Conversely, cancer cells promote fibroblast proliferations, immune cell migration and angiogenesis through signal transduction. Thus cancer microenvironment is regarded as a key contributor for epithelial-mesenchymal transition of the cancer cells, angiogenesis, cancer progression and metastasis, and development of drug resistance [1].

\footnotetext{
* Correspondence: sish.gpat@mri.tmd.ac.jp

${ }^{1}$ Department of Genomic Pathology, Medical Research Institute, Tokyo

Medical and Dental University, Tokyo, Japan

Full list of author information is available at the end of the article
}

Recently, there has been a growing interest in targeting cancer microenvironment for cancer treatment [1-4]. Inhibition of cancer stromal interaction may prevent neovascularization, invasion, and metastasis and improve anti-cancer drug delivery. For example, inhibition of Hedgehog signaling improves delivery and efficacy of gemcitabine in a mouse pancreatic cancer model [5]. However, compared to targeting driver 'mutations' which are tractable by genome-wide comparison of mutation frequency [6], exploration of driver 'interactions' is far more challenging due to the exponential number of possible interactions between proteins and lack of high-throughput methods that can quantitatively interpret the cancer-stromal interactions.

Xenograft cancers from human-derived cells grown in immune-compromised mice have been extensively used to study cancer and its microenvironment [7-12]. Xenograft cancers establish microenvironment by inducing 
mouse-derived stromal cells such as fibroblast and vascular cells, and can closely resemble the original cancer growing in a patient [8]. Given that there is approximately $15 \%$ sequence difference between human and mouse exon sequences [9], simultaneous transcriptome analysis of cancer and stroma can be achieved using RNA-Seq or species-specific microarray.

Several computational methods have been developed to analyze microarrays or RNA-Seq data from cancer xenograft mouse models. Like conventional single-species gene expression analysis, all of these methods compare gene expression profiles in two conditions, e.g. xenograft vs in cell line, or before vs after the addition of a molecule which would change the cancer-microenvironment [7, 13-15], and subsequently apply Gene Set Enrichment Analysis (GSEA) [7] or pathway analysis [13-15]. These approaches are effective in identifying gene sets or pathways contributing to the change. However, they have several limitations. First, since expression profiles of cancer cells and stromal cells are treated independently, interactions between them cannot be explicitly evaluated. Second, GSEA and pathway analysis only provide induced change as a whole, thus individual interactions cannot be evaluated. These limitations have created a bottleneck especially when the purpose is to evaluate individual interactions and to prioritize cancer-stromal interactions as the targets for cancer treatment.

To overcome such limitations, we have introduced a novel interactome analysis framework, CASTIN (CAncer-STromal INteractome analysis) for quantitative profiling of cancer-stromal interactome from RNA-Seq data using cancer xenograft mouse models. CASTIN determines direction and strength of individual transmitting signals between two interacting cells based on the expression levels of cancer and stroma. CASTIN focuses on ligand-receptor interactions because they are central to the cellular communication and, more importantly, since they involve cell surface and extracellular molecules, they are accessible by biomolecular drugs such as antibodies, peptides, and aptamers. The ligand-receptor relationships are extracted from public protein-protein interaction databases and they are manually curated. Summarization of each interaction into only three interactome evaluation indices enables us not only to quantitatively compare different interactions and to prioritize one particular interaction for clinical approach, but also to visually interpret the global cancer-stromal interactome of individual cancer and the relative importance of each interaction. To our knowledge, CASTIN is the first computational method to quantitatively evaluate cancerstromal interactions from RNA-Seq data of cancer xenograft mouse models.

We have demonstrated that CASTIN can successfully characterize the individual cancer in pancreatic cancer in terms of cancer-stromal relationships, and identify both well-known and less characterized important interactions.

\section{Results and discussion}

\section{A system for cancer-stromal interactome analysis}

Figure 1 shows the overview of the CASTIN algorithm, which consists of four main steps: (i) read assignment to human (cancer) and mouse (stroma) derived transcripts, (ii) quantification of gene expression level, (iii) integrating the gene expression with our in-house ligand-receptor database, and (iv) calculation and visualization of interactome evaluation indices for individual interactions.

In step (i), reads from cancer xenograft mouse models were assigned to RefSeq transcript sequences of human or mouse. Reads mapped to both species or to multiple genes in either species with the same number of mismatches were excluded from the subsequent analysis.

In step (ii), expression levels of each RefSeq gene in human and mouse were estimated. Although this estimation process resembles standard Transcripts Per Kilobase Million (TPM) approach [16], our strategy differs in the following two aspects:

1) CASTIN normalizes gene expression by mappable length whereas standard TPM normalizes it by transcript length. It is done by using precomputed uniquely mappable regions among human and mouse RefSeq transcript sequences. Since homologous regions are found within species or between human and mouse, some reads are not mapped uniquely and estimating expression levels without considering this may lead to inaccurate results.

2) CASTIN removes read count biases arising from regional GC content and distance from poly-A tail (Additional file 1: Figure S1), whereas standard TPM does not consider them. Although the GC bias was very slight, it has been reported in many literatures that extreme GC content leads to an uneven coverage of the transcripts in the next-generation sequencing $[17,18]$. The bias due to the distance from poly-A tail was strong in many samples. This bias is reasonable since the library construction process starts with the generation of poly-A primed cDNAs, and more fragmented the RNA is, the lower the mapped count will be in the regions farther away from the poly-A sites. The extent of RNA fragmentation differs sample by sample, but engrafted cancer tissues or cell lines would be severely influenced by this bias as they frequently show focal necrosis due to ischemia or inflammation. 


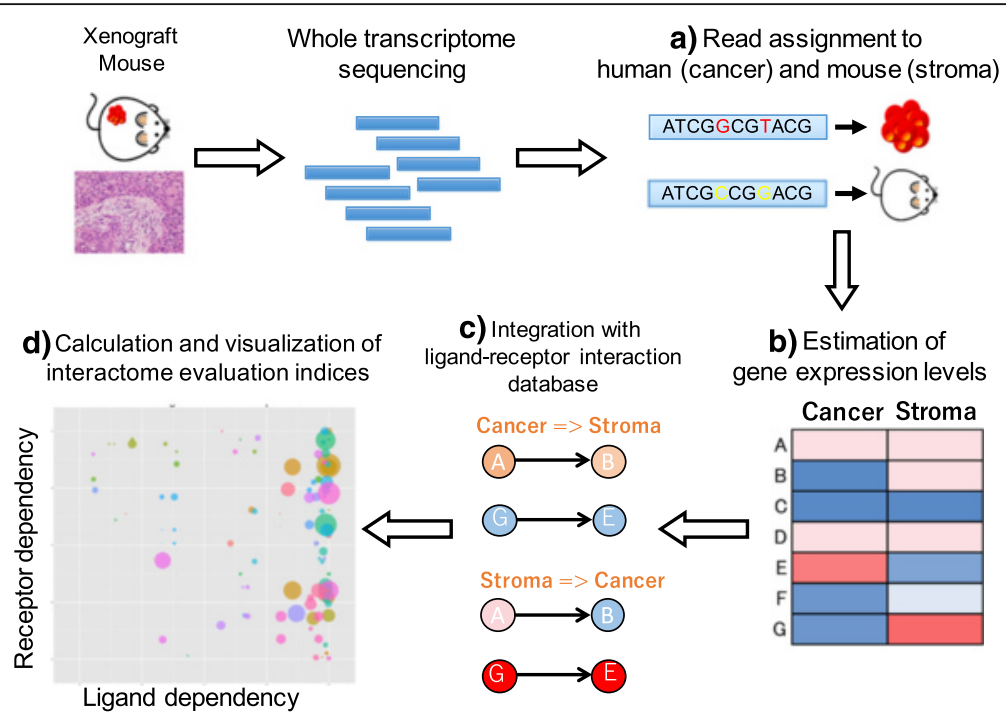

Fig. 1 workflow of the CASTIN algorithm. Total RNA extracted from cancer xenograft mouse model, which contains both human and mouse RNA, is sequenced using next generation sequencing. a Human (cancer) cell- and mouse (stromal) cell-derived transcripts are differentially assigned. $\mathbf{b}$ Accurate gene expression level is estimated using GC contents, distance from poly-A tails and originally defined mappable regions among annotated transcripts. c gene expression levels are integrated with an in-house ligand-receptor interaction database. $\mathbf{d}$ three indices (signal strength, ligand dependency, and receptor dependency) for quantitative evaluation of the interaction are calculated for the two directions of signal transduction and plot for visualization

CASTIN estimates and removes the above biases using a statistical model. Although bias correction was performed in human and mouse simultaneously, library size normalization was performed separately because cancer-to-stromal ratio in each tissue sample was different.

Then in step (iii), gene expressions were integrated to our in-house ligand-receptor interaction database. Note that ligands here included not only humoral factors but also cell surface proteins. We have created a database based on multiple protein-protein interaction databases $[19,20]$. As the establishment of high quality ligandreceptor interaction database is critical in CASTIN, researchers in the field of biology curated each interaction by carefully reviewing the original literature describing the validation experiments. Additional file 2: Table S1 lists the 628 interactions used in the current version of CASTIN.

In step (iv), three interactome evaluation indices, namely ligand dependency, receptor dependency, and signal strength, were calculated for each interaction (Fig. 2a). The evaluation indices were calculated for the two directions of signal transduction, from cancer ligand to stromal receptor and from stromal ligand to cancer receptor (hereafter referred to as $\mathrm{C}-\mathrm{S}$ direction and $\mathrm{S}-\mathrm{C}$ direction, respectively). Ligand dependency in C-S direction, defined as the expression levels of human (cancer) ligand relative to those of human (cancer) plus mouse (stroma) ligand, reflects the dependency of input signal from cancer ligand.
Receptor dependency, defined as the expression levels of mouse (stroma) receptor relative to human (cancer) plus mouse (stroma) receptors, is the counterpart of the ligand dependency and reflects the dependency of receiving signal by stromal receptor. Although these two indices are mathematically simple, combination of the two enables us to determine the major direction of signal transduction (from cancer to stroma/cancer, or from stroma to cancer/ stroma). Interactions falling into the following six zones in two-dimensional view of ligand and receptor dependency are especially relevant:

1) C-S direction (Fig. 2b):

- Zone CS1 (strong cancer cell-ligand dependency $(\geq 0.5)$, strong stromal receptor dependency $(\geq 0.5))$ : Interactions in this zone indicate that input signal is dominantly created by cancer and exclusively transmitted to stroma. The signal transduction takes place only when both cancer and stromal cells exist, and thus we call it "mutually dependent interaction".

- Zone CS2 (strong cancer cell-ligand dependency $(\geq 0.5)$, weak stromal receptor dependency $(<0.5))$ : Input signal is created by cancer and transmitted mainly to cancer itself. Thus interactions in this zone indicate cancer autoregulation.

- Zone CS3 (weak cancer cell-ligand dependency $(<0.5)$, strong stromal receptor dependency $(\geq 0.5))$ : Counterpart of zone CS2. Interactions 


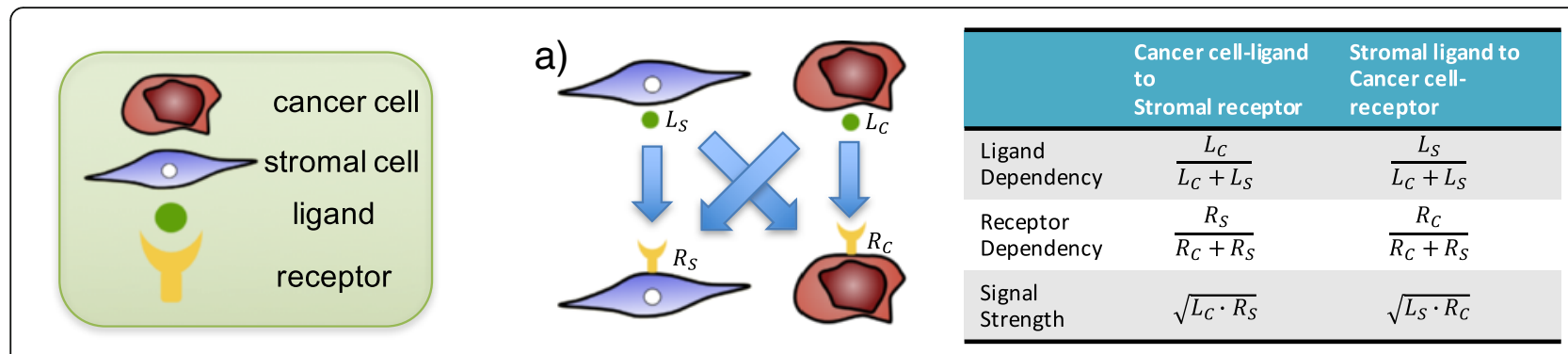

b) Cancer cell-ligand $\rightarrow$ Stromal receptor (C-S direction)

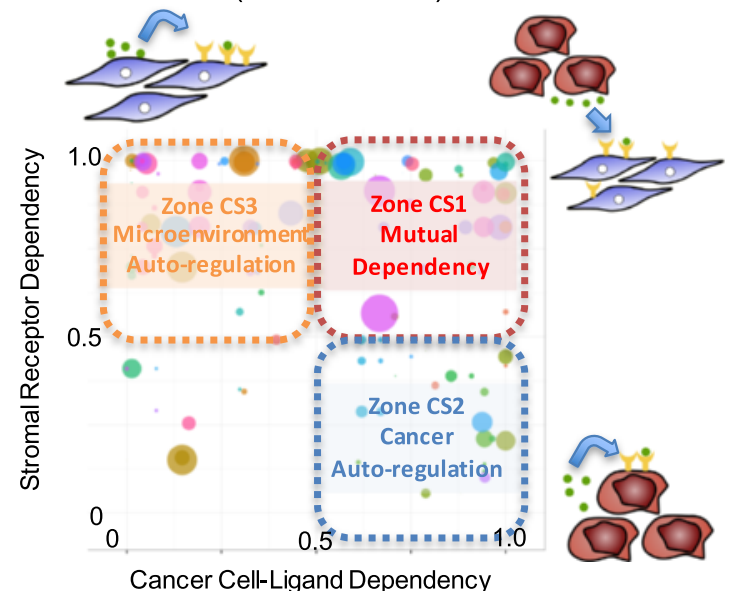

C) Stromal ligand $\rightarrow$ Cancer cell-receptor



Fig. 2 Interactome evaluation indices. a Three indices for quantitative evaluation of each interaction. Each index is calculated for two signal directions (C-S direction: cancer ligand to stromal receptor, and S-C direction: stromal ligand to cancer receptor). $\mathbf{b}$ Visualization of the evaluation indices in C-S direction. X-axis represents cancer cell-ligand dependency and y-axis represents stromal receptor dependency, and positions of interaction indicate the role of the interaction in cancer-stromal relationships, and the size of circle (proportional to log of signal strength) indicates the strength of interaction. c Visualization of the evaluation indices in S-C direction, which is the counterpart of C-S direction

in this zone indicate microenvironment autoregulation.

2) S-C direction (Fig. 2c):

- Zone SC1 (strong stromal ligand dependency $(\geq 0.5)$, strong cancer cell-receptor dependency $(\geq 0.5))$ : Interactions in this zone indicate mutually dependent interactions. It is similar to zone CS1, but the direction of signal transduction is opposite.

- Zone SC2 (strong stromal ligand dependency $(\geq 0.5)$, weak cancer cell-receptor dependency $(<0.5))$ : The direction of the signals is the same as zone CS3, but signal strength is different (See below); interactions in this zone indicate microenvironment autoregulation.

- Zone SC3 (weak stromal ligand dependency $(<0.5)$, strong cancer cell-receptor dependency $(\geq 0.5))$ : The direction of the signals is the same as zone CS2, but signal strength is different (See below); interactions in this zone indicate cancer autoregulation.
The remaining regions were designated as Zone CS4 in C-S direction (cancer cell-ligand dependency and stromal receptor dependency are both $<0.5$ ) and Zone $\mathrm{SC} 4$ in S-C direction (stromal ligand dependency and cancer cell-receptor dependency are both <0.5) for convenience.

In terms of cancer-stromal interactions, probably the most important zones among the 6 zones are CS1 and $\mathrm{SC} 1$, where the signals involve specific interaction between receptors of one cell type (i.e., cancer or stroma) and ligands from the other cell type. These "mutually dependent" or "exclusively trans-cell type" signals are potentially important therapeutic targets.

The last index, signal strength, is expressed as the geometric mean of the expression levels of cancer ligand and stromal receptor in C-S direction, or that of stromal ligand and cancer receptor in S-C direction. It is useful to remove weak and possibly non-significant interactions. As the gene expression levels were normalized so that the total expression levels roughly represent the number of mRNA molecules an average cell contains, 
the signal strength can be used to approximate the average number of mRNA molecules of the interacting genes in two cells.

Using these three indices, one can easily know the signal direction and strength of each interaction, and determine which interactions are critical, that is to say potentially druggable, in cancer-microenvironment relationships. Additionally, overall distributions of three indices, namely the interactome profile, can reflect the sample's characteristics such as stromal contribution to cancer.

As shown in Fig. 2, these indices are suitable for visualization. To help users customize visualization of the interactome, we have also developed an interactive viewer of CASTIN (http://gpatgazeza.tmd.ac.jp/CAST IN_viewer/, Additional file 3: Figure S2).

\section{Evaluation of quantification of gene expression level in CASTIN}

Unlike the conventional RNA-Seq that deals with single species, RNA-Seq of xenograft involves the separation of human/mouse reads, and we first evaluated this effect on quantification. Using uniquely mappable sequences between human and mouse reference transcripts, human and mouse-derived reads can be differentially assigned to each species. However, sequencing errors, mapping errors and nucleotide sequence variation could lead to misassignment. To investigate the rate of misassignment, cell lines derived from human cancer cells and mouse endothelial cells, both of which do not contain transcripts from any other species, were applied to CASTIN (Table 1). The misassignment rate was $0.0053-0.0124 \%$ in human cell lines and $0.0056-0.0330 \%$ in mouse cell lines (Table 2). Thus the effect of misassignment is considered to be negligible unless content of one of the

Table 1 Summary statistics of RNA-Seq for human and mouse cell lines analyzed in this study

\begin{tabular}{llllll}
\hline Sample & Species & Cell type & Cell line & Total $^{\mathrm{a}}$ & PF $^{\mathrm{b}}$ \\
\hline ExpID-112 & Human & PDAC $^{C}$ & PANC-1 & 62106630 & 43070936 \\
ExpID-114 & Human & PDAC & PK-8 & 58769786 & 44701524 \\
ExpID-115 & Human & PDAC & PK-9 & 60832395 & 44822240 \\
ExpID-116 & Human & PDAC & PK-45H & 31898332 & 29286400 \\
ExpID-117 & Human & PDAC & PK-45P & 35500162 & 32211253 \\
ExpID-118 & Human & PDAC & KLM-1 & 23673045 & 20778304 \\
ExpID-119 & Human & PDAC & MiaPaca-2 & 15255557 & 13715276 \\
ExpID-120 & Human & PDAC & Capan-1 & 41776004 & 35857527 \\
ExpID-121 & Human & PDAC & HOPE & 21063312 & 19314964 \\
ExpID-128 & Mouse & Endothelial & SVEC4-10 & 32128404 & 29780901 \\
ExpID-129 & Mouse & Endothelial & IP-1B & 34391406 & 31548417 \\
\hline
\end{tabular}

${ }^{a}$ Total Number of reads

${ }^{b}$ Number of reads passing Illumina's filter

'Pancreas ductal adenocarcinoma species is extremely small, where interactome analysis is essentially unsuitable. In the CASTIN system, we have employed a deterministic approach when assigning sequencing reads into each species, and as a result very accurate assignment was achieved. However probabilistic approaches such as Expectation-Maximization algorithm [16] could improve classification performance further.

CASTIN discards reads derived from human or mouse when they were mapped on unmappable (identical sequence between human and mouse) regions, which could result in inaccurate gene expression levels. However, as our algorithm considers "mappable" length instead of transcript length, the effect should be minimal as far as the read coverage within gene is close to uniform after removing the effect of distance from polyA tail and regional GC content. To investigate the effect, gene expression levels of human cell lines using mappable regions of only human and both human and mouse were compared. Gene expression levels of mouse cell lines were also evaluated in a same manner. Table 2 shows the Pearson correlation between the two conditions for each sample. Very strong correlations in both human and mouse indicate that correction of the effect of mappable length worked well.

Additionally, we have performed RNA-seq analysis of artificial mixtures of human (PANC-1 cell line) and mouse (SVEC4-10 cell line) total RNA to evaluate the reproducibility of gene expression quantitation under various tumor-stromal ratios (human content: $0,25,50$, 75, and $100 \%$ ) (Additional file 4: Table S2, Additional file 5: Figure S3). In both human and mouse, the estimated gene expression levels had very high correlation (human; 0.97-0.99, mouse; 0.94-0.99) on the identical line regardless of human-to-mouse ratios. Because mixed RNA samples were sequenced with Illumina GAIIx and pure human or mouse samples were sequenced with HiSeq2000, the correlations slightly decreased when comparing the results from different sequencing platforms. Nonetheless, these results demonstrated the highly reliable and reproducible gene expression quantitation under various conditions with different tumor-stromal ratios.

Finally, we have compared the results obtained from CASTIN with protein expression determined by immunohistochemistry. We applied CASTIN to the dataset of pancreas ductal adenocarcinoma (PDAC) consisting of 8 xenograft samples from different PDAC cell lines (Table 3). We have selected FABP5/Fabp5 gene for analysis because it showed various gene expression ratios between human and mouse in PDAC xenograft samples (human-to-mouse ratios ranged from 0.028 to 3.4, Additional file 6: Figure S4a), and also the antibody with human/mouse cross-reactivity and FFPE compatibility was commercially available. FABP5/Fabp5 stained cancer 
Table 2 Effect of mappable regions between human and mouse on estimated gene expression levels

\begin{tabular}{|c|c|c|c|c|c|c|}
\hline Sample & Species & Cell line & Human $^{a}$ & Mouse $^{b}$ & Error $(\%)^{c}$ & Correlation $^{\mathrm{d}}$ \\
\hline ExplD-112 & Human & PANC-1 & 32194053 & 1821 & 0.0057 & 0.9992012 \\
\hline ExplD-114 & Human & PK-8 & 34975755 & 1867 & 0.0053 & 0.9998013 \\
\hline ExplD-115 & Human & PK-9 & 32170808 & 3484 & 0.0108 & 0.9997474 \\
\hline ExplD-116 & Human & PK-45H & 17278113 & 1331 & 0.0077 & 0.9998448 \\
\hline ExplD-117 & Human & PK-45P & 18986898 & 1243 & 0.0065 & 0.9997115 \\
\hline ExplD-118 & Human & KLM-1 & 8950786 & 799 & 0.0089 & 0.999563 \\
\hline ExplD-119 & Human & MiaPaca-2 & 2251017 & 1192 & 0.0530 & 0.9997128 \\
\hline ExplD-120 & Human & Capan-1 & 2829135 & 2195 & 0.0776 & 0.9995722 \\
\hline ExplD-121 & Human & HOPE & 8930382 & 1110 & 0.0124 & 0.9996743 \\
\hline ExplD-128 & Mouse & SVEC4-10 & 1052 & 18625926 & 0.0056 & 0.9987255 \\
\hline ExplD-129 & Mouse & IP-1B & 6508 & 19707229 & 0.0330 & 0.9988737 \\
\hline
\end{tabular}

${ }^{a}$ Number of reads assigned to human by the CASTIN system

${ }^{b}$ Number of reads assigned to mouse by the CASTIN system

cMisassignment rate (\%)

'Pearson's correlation coefficient between gene expression levels of human (mouse) cell lines using mappable regions of only human (mouse) and both human and mouse

cells homogeneously except for KLM-1 (Additional file 6: Figure S4b); stromal cells were stained homogeneously or heterogeneously in each sample, reflecting the different cell populations that comprise stroma (e.g. fibroblasts, leukocytes, vascular endothelial cells). Although immunohistochemistry is not strictly quantitative, the relative staining intensity between human cancer and mouse stromal cells well reflected the human to mouse ratio of RNA-Seq reads from the same xenograft tumor.

\section{Interactome profiles correlate to histology of pancreatic cancer}

To demonstrate that interactome profiles of CASTIN correlate histology of cancer and stroma, we applied CASTIN to the dataset of PDAC consisting of 8 xenograft samples from cell lines (Table 3). PDAC was chosen because one of its defining features is the presence of extensive desmoplasia and recent studies have shown that cancer-stromal interaction plays a key role in PDAC development [21].

We hypothesized that the stronger the desmoplastic reaction is, the stronger the signals in zones CS1 (cancer to stroma), CS3 (stroma to stroma), SC1 (stroma to cancer), or SC2 (stroma to stroma) will be. Thus we counted the number of interactions in zone CS1 or CS3, and SC1 or SC2 (Fig. 3a). It clearly showed that Miapaca-2 has weaker signals related to stroma in both directions compared to others such as Capan-1. Such tendency can also be easily seen visually in interactome profiles (Fig. 3b). As expected, Capan-1 shows desmoplastic histology with rich stroma, whereas Miapaca-2 shows medullary histology with poor stroma content (Fig. 3c), which is atypical in PDAC.

These results demonstrate that the global interactome profile reflects the actual cancer-stromal interaction in vivo well, and CASTIN is useful to characterize cancers with respect to cancer-stromal relationships.

Table 3 Summary statistics of RNA-Seq for PDAC xenograft models analyzed in this study

\begin{tabular}{|c|c|c|c|c|c|c|}
\hline Sample & Cell line & Total $^{a}$ & $\mathrm{PF}^{\mathrm{b}}$ & Human $^{c}$ & Mouse $^{d}$ & Mouse (\%) \\
\hline ExplD-88 & KLM-1 & 40315040 & 36210281 & 14306947 & 915402 & 6.01 \\
\hline ExplD-89 & Capan-1 & 42088225 & 37433177 & 18147400 & 2041946 & 10.11 \\
\hline ExplD-90 & PANC-1 & 42912362 & 38177843 & 18700371 & 2007413 & 9.69 \\
\hline ExplD-91 & PK-1 & 36841310 & 33534549 & 14071808 & 5663160 & 28.70 \\
\hline ExplD-92 & PK-8 & 37239118 & 33971461 & 15024350 & 2902408 & 16.19 \\
\hline ExplD-93 & PK-45P & 38612205 & 35070083 & 11713564 & 9796740 & 45.54 \\
\hline ExplD-94 & PK-9 & 40328852 & 36514269 & 16929209 & 2015939 & 10.64 \\
\hline ExplD-95 & MiaPaca-2 & 39641561 & 35751983 & 20439739 & 2037175 & 9.06 \\
\hline
\end{tabular}

${ }^{\mathrm{a}}$ Total Number of reads

${ }^{b}$ Number of reads passing Illumina's filter

${ }^{c}$ Number of reads assigned to human by the CASTIN system

${ }^{\mathrm{d}}$ Number of reads assigned to mouse by the CASTIN system

e Percentage of mouse reads, expressed by $d /(c+d)$ 


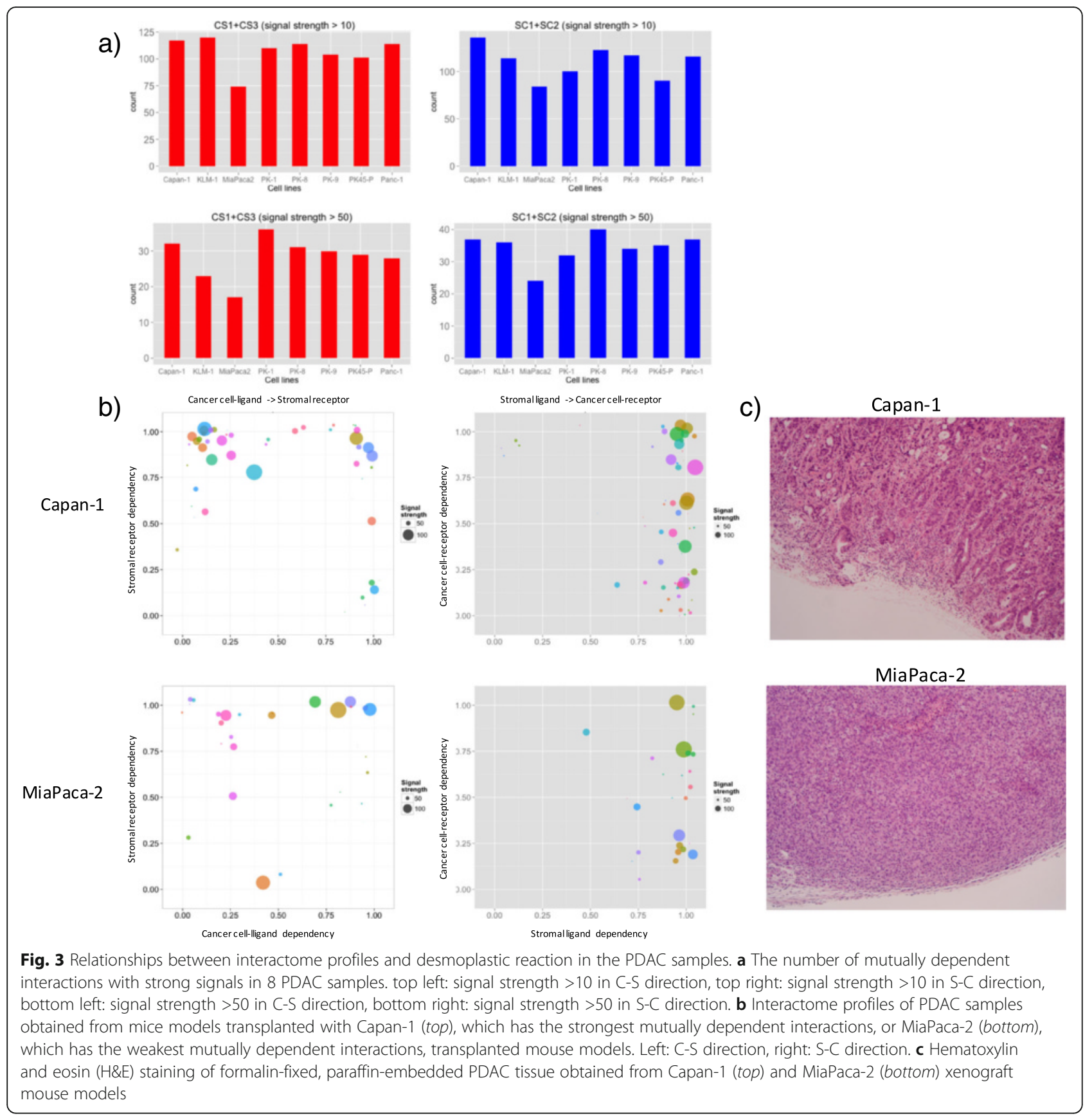

\section{CASTIN detects known interactions targeted by existing} drugs and less-characterized druggable interactions

Next we have investigated profiles of interactions targeted by existing drugs. In particular, we focused on the interactions involving kinases because kinase inhibitors are currently the most successful molecular targeted drugs [22]. PDAC dataset used in the previous section was also analyzed in this section. To summarize eight interactome profiles into a single profile, each averaged evaluation index for every interaction was used (Fig. 4). As shown in Fig. 4a and b, interactions inhibited by currently available molecular targeted cancer drugs such as $\mathrm{HGF}^{1}-\mathrm{MET}^{2}$ (e.g. Tivantinib), EGF ${ }^{3}-\mathrm{EGFR}^{4}$ (e.g. Erlotinib), VEGFA ${ }^{5}-\mathrm{KDR}^{6}$ (e.g. Ramucirumab) and VEGFB $^{7}$-FLT1 ${ }^{8}$ (e.g. Pazopanib) tend to have strong signals and reside around zones SC1 and CS1. While the receptors of the former two interactions (MET and EGFR) are mainly expressed in cancer cells [23, 24] and promote cancer cell proliferation, the receptors of the latter two interactions (KDR and FLT1) are mainly expressed in vascular endothelial cells in microenvironment [25], and drugs targeting these molecules inhibit 



Fig. 4 Interactome profiles of PDAC samples. Interactome profiles of kinases in (a) C-S direction and (b) S-C direction, GPCR in (c) C-S direction and (d) S-C direction, non-GPCR, non-Kinase in (e) C-S direction, and (f) S-C direction. Interactions targeted by molecular targeted drugs on the market are indicated by arrows

cancer neovascularization. Based on the signal direction trend of these successfully marketed drugs, the interactions in zones SC1 and CS1 or "mutually dependent" interactions are suggested to be prioritized targets of therapeutic intervention.

Hedgehog signaling between cancer and stroma is known to induce desmoplastic reaction in stroma, and the effect of its inhibition is applied in clinical trials [26] as the improvement of anti-cancer drug delivery was observed in PDAC mouse model [5]. Three hedgehogrelated ligand-receptor interactions are included in our database: sonic hedgehog (SHH)- patched 1 (PTCH1), indian hedgehog (IHH)-PTCH1, and IHH-patched 2 (PTCH2). In our analysis, all 3 hedgehog-related interactions were found in zone CS1 with strong signals in Capan-1 xenograft mouse (Additional file 7: Figure S5), suggesting the importance of this signal in pancreatic cancer. However, the signal strength and direction are highly variable between the 8 pancreatic cancer cells. It seems that that the contribution of hedgehog signaling differs among each PDAC sample and that even though hedgehog inhibitor is ineffective for pancreatic cancer as a total [26], personalized medicine may be achieved by developing companion diagnostics to stratify patients 
based on the contribution of hedgehog signaling in their cancer tissues. Based on the results above, we have searched for less-characterized druggable interactions, which have strong signals and reside in zone CS1 or $\mathrm{SC} 1$, in PDAC data. Mutually dependent interactions were extracted in both directions from PDAC data, and interactions with ligand dependency $>0.75$, receptor dependency $>0.75$, and signal strength $>50$ were selected. A literature survey of these interactions were summarized in Table 4.

In C-S direction, semapholin 3C (SEMA3C) neuropilin-1 (NRP1) interaction (Additional file 8: Figure S6a) has the strongest signal. NRP1 is a transmembrane receptor for SEMA3C and vascular endothelial cell growth factor 165 (VEGF165) [27]. NRP1 promotes angiogenesis through binding to VEGF, but mediates antiangiogenic effects by interacting with semapholin 3B and $3 \mathrm{~F}$, the other class 3 semaphorins, indicating that they act as the antagonists that block NRP1 binding to

Table 4 Mutually dependent interactions with strong signals in PDAC dataset

\begin{tabular}{|c|c|c|c|c|}
\hline ligand & receptor & direction & $\begin{array}{l}\text { signal } \\
\text { strength }^{\mathrm{a}}\end{array}$ & $\begin{array}{l}\text { possible relevance for } \\
\text { cancer-stromal interactions }\end{array}$ \\
\hline SEMA3C & NRP1 & $C-S$ & 101.5 & $\begin{array}{l}\text { SEMA3C induces growth } \\
\text { and migration of endothelial } \\
\text { cells [29], suggesting its } \\
\text { angiogenic role; NRP1 also } \\
\text { causes desmoplastic reaction } \\
\text { in cancer [30]. }\end{array}$ \\
\hline WNT7B & GPC3 & C-S & 50 & $\begin{array}{l}\text { In hepatocellular carcinoma, } \\
\text { GPC3 promotes cancer cell } \\
\text { growth by Wnt signaling } \\
\text { including WNT7B [53]. }\end{array}$ \\
\hline COL1A2 & CD44 & $\mathrm{S}-\mathrm{C}$ & 753 & $\begin{array}{l}\text { CD44 expressed in PDAC } \\
\text { regulates its invasion [54]. }\end{array}$ \\
\hline COL1A1 & CD44 & $\mathrm{S}-\mathrm{C}$ & 668.4 & $\begin{array}{l}\text { CD44 expressed in PDAC } \\
\text { regulates its invasion [54]. }\end{array}$ \\
\hline FN1 & ITGA3 & $\mathrm{S}-\mathrm{C}$ & 496.6 & Not reported \\
\hline COL1A2 & ITGA2 & $\mathrm{S}-\mathrm{C}$ & 348.4 & $\begin{array}{l}\text { a } 2 \beta 1 \text { integrin-mediated } \\
\text { adhesion on type I collagen } \\
\text { promotes the malignant } \\
\text { phenotype in PDAC [32]. }\end{array}$ \\
\hline COL1A1 & ITGA2 & $\mathrm{S}-\mathrm{C}$ & 341.1 & $\begin{array}{l}\text { a } 2 \beta 1 \text { integrin-mediated } \\
\text { adhesion on type I collagen } \\
\text { promotes the malignant } \\
\text { phenotype in PDAC [32]. }\end{array}$ \\
\hline FN1 & ITGB6 & $\mathrm{S}-\mathrm{C}$ & 180.8 & $\begin{array}{l}\text { Promotes breast cancer } \\
\text { invasion [55]. }\end{array}$ \\
\hline SEMA4D & PLXNB1 & $\mathrm{S}-\mathrm{C}$ & 63.4 & $\begin{array}{l}\text { Promotes cancer cell } \\
\text { motility in PDAC [33]. }\end{array}$ \\
\hline SFRP1 & FZD6 & $\mathrm{S}-\mathrm{C}$ & 62 & $\begin{array}{l}\text { FZD6 overexpressed in } \\
\text { several cancers [56]; SFRP1 } \\
\text { is a Wnt antagonist. }\end{array}$ \\
\hline IGF1 & IGF1R & S-C & 54.5 & $\begin{array}{l}\text { IGF1R induces PDAC growth } \\
\text { and metastasis [57]. }\end{array}$ \\
\hline
\end{tabular}

${ }^{a} \mathrm{C}-\mathrm{S}$ : signal transduction from cancer cell-ligand to stromal receptor $\mathrm{S}-\mathrm{C}$ : signal transduction from stromal ligand to cancer cell-receptor
VEGF [28]. Meanwhile, SEMA3C was found to induce growth and migration of endothelial cells [29], which suggests its angiogenic role in cancer. Interestingly, NRP1 also causes desmoplastic reaction in cancer; genetic depletion or antibody neutralization of NRP1 from stromal myofibroblast was shown to reduce cancer growth and fibronectin fibril assembly in vivo [30], although the ligand of NRP1 was not investigated in the report. Considering pronounced desmoplasia of PDAC, our data suggests that NRP1 may contribute to desmoplastic reaction in PDAC.

In $\mathrm{S}-\mathrm{C}$ direction, interactions including collagens and fibronectins have strong signals. This is reasonable because stromal cells (e.g. fibroblast) highly express extracellular matrix molecules, such as collagen, fibronectin, and laminin [31]. A notable example among these interactions is the one between type I collagen (COL1A1 and COL1A2) and alpha-2 integrin (ITGA2). $\alpha 2 \beta 1$ integrinmediated adhesion on type I collagen has been reported to promote the malignant phenotype in PDAC [32].

Other than collagens and fibronectins, semapholin 4D (SEMA4D) and plexin B1 (PLXNB1) interaction has the strongest signal in zone $\mathrm{SC} 1$ (Additional file 8: Figure S6 b). It has been reported that binding of SEMA4D to PLXNB1 promotes cancer cell motility in PDAC [33]. Additionally, increased expression of both SEMA4D and PLXNB1 was associated with poor prognosis [34]. Immunohistochemical analysis showed that SEMA4D was predominantly expressed in the cancer stroma and PLXNB1 was predominantly expressed in cancer epithelial cells in PDAC [34], which is compatible with the evaluation indices at transcript level in our study.

Previous studies have suggested the importance of semapholin signaling in cancer-microenvironment [29, 33-35]. However, its relative importance among all the cancer-stromal interactions has not been quantitatively evaluated due to the lack of methods. Our interactome analysis using CASTIN suggested that semapholin signaling, especially SEMA4D and SEMA3C, plays particularly important roles and these molecules/proteins are potential targets in pancreatic cancer.

Although many researchers have investigated various cancer-stromal interactions which are potential therapeutic targets, prioritization among multiple interactions have not been done as these interactions have been evaluated only individually in most cases. One of the advantages of CASTIN is that using three evaluation indices we can compare multiple interactions based on their role in cancer-stromal interactions and identify which interactions are vital and should be inhibited for clinical approach. Importantly, our ligand-receptor database contains interactions involving extracellular and cell surface proteins, which are easily accessible by biomolecular drugs (large molecules such as antibodies). It is well 
known that biomolecular drugs greatly expands target interactions/proteins outside of classical druggable proteins, that could be targeted by small molecule drugs. In our study, more than half $(431 / 628)$ of the interactions identified by CASTIN do not involve kinases or GPCRs, which are typical "druggable" proteins. (Fig. 4e, f).

\section{Contribution of "functional modules" in cancer-stromal interactome}

CASTIN assigns each ligand-receptor interaction into zones which reflect the direction of signal transduction in cancer-stromal relationships. As genes with similar function are expected to behave similarly, analyzing interactions having similar functions collectively using CASTIN will help us to understand the role of "functional modules" in cancer-stromal relationships. Hence we have investigated the interactome profiles of genes with various molecular functions. Here again we applied CASTIN to the PDAC and the averaged interactome profile was analyzed. We defined an interaction having molecular function when either ligand or receptor in the interaction belonged to Gene Ontology (GO) functional categories. Functional modules with characteristic pattern among 44253 GO categories were shown in Figs. 5 and 6.

Functional modules located in zone CS1 and CS2 indicate that only cancer cells secrete ligands (Fig. 7a). Actually, however, most interactions in this type are predominantly located in CS2, which indicates autoregulation of cancer cells. Interestingly, all the functional modules are related to ephrins and Eph receptors. The most representative example is 'ephrin receptor binding' (Fig. 7a). Eph-ephrin complexes produce bidirectional signals and affect cancer growth, invasiveness and metastasis [36]. For example, EPHA2 and EPHB4 within ephrin family are widely expressed in cancer cells, and their expression has been linked to cancer progression [36]. Downregulation of EPHA2 or EPHB4 expression with siRNAs or antisense oligonucleotides results in inhibition of malignant cell behavior in culture and cancer growth in vivo [36]. This is in line with our result that both EPHB4-EFNB2 and EPHA2-EFNA1 interactions have strong signals (Fig. 7a).

Functions located in zone CS1 and CS3 are clustered into two groups: functions related to vascular endothelial growth factor (VEGF), platelet-derived growth factor (PDGF), and semaphorins, and functions related to transforming growth factor beta (TGF $\beta$ ). The VEGF and PDGF related interactions preferentially located in zone CS1 and CS3, which is indicative of stromal cells receiving strong signal from cancer or microenvironment (Fig. 7b). Meanwhile, the TGF $\beta$ related interactions predominantly located in zone CS3, which is indicative of microenvironment autoregulation. VEGF and PDGF signaling contribute to angiogenesis in PDAC [37], and
VEGF expression in cancer and PDGF receptor expression in stroma are associated with poor prognosis in several types of cancers including PDAC [38, 39]. Semaphorins are important regulators in cancer cells [35], and high expression of SEMA4D was associated with poor survival in PDAC [34] as mentioned in the previous section. TGF $\beta$ has the ability to induce fibroblast proliferation in PDAC [40], and autocrine TGF $\beta$ signaling regulates myofibrogenesis in carcinoma-associated fibroblasts during fibrosis in breast cancer [41], indicating that stromal cells could be a source of TGF $\beta$ in other cancer. In particular, our analysis suggested that TGF $\beta 1$ and its receptor TGF $\beta 1$ receptor 1 (T $\beta R 1)$ produce strong signal (Fig. 7c). Indeed, TGF $\beta 1$, by interacting $\mathrm{T} \beta \mathrm{R} 1$, directly elicits desmoplastic reaction in pancreatic cancer [42]. Many T $\beta R 1$ inhibitors have been developed to improve chemopenetration, and among them, SD-208 reduced fibrosis in cancer microenvironment [43]. Another interaction with strong signaling is TGF 1 -endoglin (EGN). Endoglin is a cell-surface glycoprotein and is part of the TGF $\beta$ receptor complex [44]. It also has a crucial role in angiogenesis and is abundantly expressed in vascular endothelial cells at sites of active angiogenesis [44]. In pancreatic cancer tissues, endoglin is highly expressed in endothelial cells forming small capillarylike vessels [45].

GO functional modules predominantly located in zone SC1 and SC2 (Fig. 6a) consistently have strong stromal ligand dependencies, indicating that both cancer and stromal cells are receiving strong signal exclusively from stromal cells. We have found that most of them are related to extracellular matrix such as 'extracellular matrix structural constituent' (Fig. 7d). A notable example is the interaction between type I collagen (COL1A1 and COL1A2) and alpha-2 integrin (ITGA2), which we referred in the previous section.

Functional modules located in zone SC1 and SC3 have very low signal strength (Fig. 6b). Top 2 categories 'excitatory synapse' and 'negative regulation of protein kinase $\mathrm{B}$ signaling' has relatively strong signals. However, interactions contributing to the strong signals are related to FN1 and laminin, both of which are related to extracellular matrix and predominantly located in SC1. Thus the we do not discuss the functional modules in this category further.

As shown above, functional modules preferentially located in each zone have distinctive profile reflecting the role in cancer-stromal interactions. These results demonstrate that our interactome analysis reflects molecular functions and useful to prioritize important interactions between cancer and stroma.

\section{Conclusions}

We have developed CASTIN that can quantitatively assess cancer-stromal interactome using RNA-Seq data 


\begin{tabular}{|c|c|c|c|c|c|c|}
\hline \multicolumn{7}{|l|}{ a) } \\
\hline & & & zones in & direction & & \\
\hline GO accession & GO name & |mean signal & $\frac{\mathrm{CS3}}{\mathrm{CS4}}$ & $\frac{\mathrm{CS1}}{\mathrm{CS} 2}$ & qvalue & |Ephrin \\
\hline GO:0046658 & anchored component of plasma membrane & 18.4 & $\frac{1}{1}$ & $\begin{array}{ll}4 \\
10\end{array}$ & $9.85 \mathrm{E}-02$ & \\
\hline GO:0033598 & mammary gland epithelial cell proliferation & 16.6 & $\frac{1}{0}$ & $\frac{0}{10}$ & $1.96 \mathrm{E}-01$ & \\
\hline GO:0070848 & response to growth factor & 16.6 & $\frac{1}{0}$ & $\frac{0}{10}$ & $1.96 \mathrm{E}-01$ & \\
\hline GO:0003199 & endocardial cushion to mesenchymal transition involved in heart valve formation & 16.4 & $\frac{0}{1}$ & $\frac{1}{10}$ & $1.42 E-01$ & \\
\hline GO:0014028 & notochord formation & 14.4 & $\frac{0}{1}$ & $\frac{1}{18}$ & $2.83 E-03$ & \\
\hline GO:0043535 & regulation of blood vessel endothelial cell migration & 14.4 & $\frac{2}{1}$ & $\frac{1}{12}$ & $3.02 E-02$ & \\
\hline GO:0033628 & regulation of cell adhesion mediated by integrin & 11.9 & 2 & $\frac{1}{22}$ & $5.33 E-03$ & \\
\hline GO:0072178 & nephric duct morphogenesis & 11.9 & 0 & $\frac{1}{2}$ & $4.21 E-04$ & \\
\hline GO:0046875 & ephrin receptor binding & 11.8 & 0 & 6 & $1.38 E-06$ & \\
\hline
\end{tabular}

b)

\begin{tabular}{|c|c|c|c|c|c|c|c|c|}
\hline \multirow[b]{2}{*}{ GO accession } & \multirow[b]{2}{*}{ GO name } & \multirow[b]{2}{*}{ mean signal } & \multicolumn{2}{|c|}{ zones in C-S direction } & \multirow[b]{2}{*}{ qvalue } & \multirow[b]{2}{*}{ VEGF } & \multirow[b]{2}{*}{ TGFB } & \multirow[b]{2}{*}{ BMP SEMA } \\
\hline & & & $\operatorname{cs3}$ & CS1 & & & & \\
\hline GO:0038085 & vascular endothelial growth factor binding & 43.7 & 6 & 6 & 2.49E-03 & & & \\
\hline GO:0038084 & vascular endothelial growth factor signaling pathway & 37.2 & 5 & 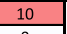 & $5.88 \mathrm{E}-04$ & & & \\
\hline $\mathrm{GO}: 0048846$ & axon extension involved in axon guidance & 36.8 & 2 & $\frac{9}{0}$ & 4.10E-03 & & & \\
\hline GO:0034713 & type I transforming growth factor beta receptor binding & 36.6 & 10 & $\frac{2}{0}$ & $2.49 \mathrm{E}-03$ & & & \\
\hline GO:0048842 & positive regulation of axon extension involved in axon guidance & 35.7 & $\frac{2}{0}$ & $\frac{11}{0}$ & $1.64 \mathrm{E}-03$ & & & \\
\hline GO:0005902 & microvillus & 35.6 & $\frac{12}{0}$ & $\frac{1}{0}$ & $1.64 E-03$ & & & \\
\hline GO:0034714 & type III transforming growth factor beta receptor binding & 35.3 & $\frac{12}{0}$ & $\frac{0}{0}$ & $2.49 \mathrm{E}-03$ & & & \\
\hline GO:0010936 & negative regulation of macrophage cytokine production & 35.3 & $\frac{12}{0}$ & $\begin{array}{ll}0 \\
0\end{array}$ & $2.49 E-03$ & & & \\
\hline GO:0043117 & positive regulation of vascular permeability & 34.5 & $\frac{9}{0}$ & $\frac{3}{0}$ & $2.49 \mathrm{E}-03$ & & & \\
\hline GO:0005021 & vascular endothelial growth factor-activated receptor activity & 33.6 & $\frac{9}{0}$ & $\frac{11}{0}$ & $5.39 E-05$ & & & \\
\hline
\end{tabular}

Fig. 5 GO categories predominantly located in CS1, CS2 and CS3. a Top 9 GO categories predominantly located in CS1 and CS2. $10^{\text {th }}$ GO category was removed because its mean signal strength was < 10. b Top 10 GO categories were predominantly located in CS1 and CS3. GO categories were sorted by the percentage of interactions located in the corresponding zones, and then sorted by the mean signal intensity of all the interactions in the GO category. The number of interactions in each zone (CS1, CS2, CS3, and CS4) is shown in 'zones in C-S direction' and the intensity of red color is proportional to the number of interactions. False discovery rate ( $q$-value) is shown in 'qvalue'. Representative genes or gene families, which appear in at least $2 \mathrm{GO}$ categories and signal strength $>10$ for at least 1 interaction, is depicted by green boxes. 'Mean signal' refers to the mean signal strength of the interactions in the GO category. Four boxes in 'zones in C-S direction' indicate the number of interactions within each zone. Zones CS1 (top right), CS2 (bottom right), CS3 (top left), and CS4 (bottom left)

from cancer xenograft mouse models. Key aspects of CASTIN are high quality, manually curated ligand-receptor database and three evaluation indices, which are easy to interpret and also suitable for visualization. By showing some examples using PDAC dataset, we have shown that these unique features provide researchers with useful information for interpreting cancer-stromal interactome such as a role of each interaction in signal transduction between cancer and stromal cells thus enables prioritization of drug target, and characterization of individual cancer sample in terms of cancer-microenvironment interactions. So far, there are no comparative methods that can perform comprehensive analyses like CASTIN. We have also made the CASTIN software and its viewer publicly available. The software accepts FASTQ (single-end and paired-end) files of RNA-Seq from xenograft samples.
The CASTIN method could also be used to analyze xenograft models of other human cancer types. In the future this method might even be used to identify the cancer-stroma interactome in PDX models and to apply personalized medicine to each patient depending on the many interactions identified. We note that CASTIN is not applicable to early passage PDX models in which human stroma can still be detected, as it would lead to stromal contamination in estimated cancer gene expression levels.

There are several limitations in CASTIN. First, some ligand-receptor relationships may have been left out from our interaction database as our curation process and the database (KEGG or HPRD) only included relationships with adequate experimental evidences. Newly reported protein interactions with sufficient evidence 


\begin{tabular}{|c|c|c|c|c|c|c|c|c|}
\hline \multicolumn{9}{|l|}{ a) } \\
\hline \multirow{3}{*}{ GO accession } & \multirow{3}{*}{ GO name } & \multirow{3}{*}{ mean signal } & \multicolumn{2}{|c|}{ zones in S-C direction } & \multirow{3}{*}{ qvalue } & \multirow{3}{*}{ FN1 } & \multirow{3}{*}{ Collagen } & \multirow{3}{*}{ Laminin } \\
\hline & & & $\mathrm{SC3}$ & SC1 & & & & \\
\hline & & & $\mathrm{SC4}$ & $\mathrm{SC2}$ & & & & \\
\hline GO:0005201 & extracellular matrix structural constituent & 222.3 & $\begin{array}{ll}0 \\
0\end{array}$ & $\frac{7}{5}$ & $2.49 \mathrm{E}-03$ & & & 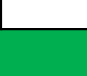 \\
\hline GO:0005518 & collagen binding & 175.5 & $\begin{array}{ll}0 \\
0\end{array}$ & $\begin{array}{c}9 \\
13 \\
\end{array}$ & $5.88 \mathrm{E}-04$ & & & \\
\hline GO:0030214 & hyaluronan catabolic process & 164.8 & $\begin{array}{l}0 \\
0\end{array}$ & $\frac{4}{7}$ & 4.10E-03 & & & \\
\hline GO:0071288 & cellular response to mercury ion & 124.1 & 0 & $\frac{3}{9}$ & $2.49 \mathrm{E}-03$ & & & \\
\hline GO:0007044 & cell-substrate junction assembly & 122.6 & $\begin{array}{l}0 \\
0\end{array}$ & $\frac{3}{15}$ & $1.64 \mathrm{E}-03$ & & & \\
\hline GO:0071380 & cellular response to prostaglandin E stimulus & 111.8 & $\frac{0}{0}$ & $\frac{5}{9}$ & $1.64 \mathrm{E}-03$ & & & \\
\hline GO:0036120 & cellular response to platelet-derived growth factor stimulus & 108.4 & $\frac{1}{0}$ & $\frac{4}{10}$ & $2.49 \mathrm{E}-03$ & & & \\
\hline GO:0007613 & memory & 93.6 & $\begin{array}{ll}0 \\
0\end{array}$ & $\begin{array}{c}3 \\
12\end{array}$ & $2.49 \mathrm{E}-03$ & & & \\
\hline GO:0045727 & positive regulation of translation & 84.4 & $\begin{array}{ll}0 \\
0\end{array}$ & $\begin{array}{ll}4 \\
7\end{array}$ & $2.49 \mathrm{E}-03$ & & & \\
\hline GO:0071062 & alphav-beta3 integrin-vitronectin complex & 66.7 & $\begin{array}{ll}0 \\
0\end{array}$ & $\begin{array}{ll}2 \\
12 \\
\end{array}$ & $5.39 \mathrm{E}-05$ & & & \\
\hline b) & & & & & & & & \\
\hline & & & & \begin{tabular}{|l|l|} 
zones in $\mathrm{S}$ \\
\end{tabular} & S-C direction & & & \\
\hline GO accession & GO name & & mean signal & \begin{tabular}{|l|} 
SC3 \\
SC4 \\
\end{tabular} & \begin{tabular}{|l|}
$\mathrm{SC} 1$ \\
$\mathrm{SC2}$ \\
\end{tabular} & qvalue & FGF & Ephrin \\
\hline GO:0060076 & excitatory synapse & & 41.1 & $\begin{array}{l}4 \\
0\end{array}$ & $\frac{9}{0}$ & $2.76 \mathrm{E}-03$ & & \\
\hline GO:0051898 & negative regulation of protein kinase B signaling & & 22.9 & $\frac{11}{0}$ & $\begin{array}{ll}3 \\
0\end{array}$ & $1.70 \mathrm{E}-03$ & & \\
\hline GO:0030900 & forebrain development & & 13.5 & $\frac{10}{1}$ & $\frac{10}{0}$ & $7.31 E-04$ & & \\
\hline GO:0072178 & nephric duct morphogenesis & & 11.9 & $\frac{22}{1}$ & $\frac{1}{0}$ & $2.08 \mathrm{E}-04$ & & \\
\hline $\begin{array}{l}\text { Fig. } 6 \mathrm{GO} \text { cate } \\
\text { categories pre } \\
\text { are processed } \\
\text { (top right), SC2 }\end{array}$ & $\begin{array}{l}\text { lantly located in SC1, SC2 and SC3. a Top } 10 \text { GO catego } \\
\text { ed in SC1 and SC3. Fifth to } 10^{\text {th }} \text { GO categories were ren } \\
\text { s in Fig. 5. Four boxes in 'zones in S-C direction' indicate } \\
\text { C3 (top left), and SC4 (bottom left) }\end{array}$ & $\begin{array}{l}\text { ries pred } \\
\text { noved be } \\
\text { the num }\end{array}$ & $\begin{array}{l}\text { ominantly } \\
\text { cause the } \\
\text { ber of int }\end{array}$ & $\begin{array}{l}\text { located } \\
\text { ir mean } \\
\text { teraction }\end{array}$ & $\begin{array}{l}\text { in } \mathrm{SC} 1 \text { a } \\
\text { signal st } \\
\text { ns within }\end{array}$ & $\begin{array}{l}\text { and SC2. } \\
\text { trength } v \\
\text { each zO }\end{array}$ & $\begin{array}{l}\text { b Top } \\
\text { was }<10 \\
\text { one. Zor }\end{array}$ & $\begin{array}{l}4 \mathrm{GO} \\
\text { Data } \\
\text { es SC1 }\end{array}$ \\
\hline
\end{tabular}

will be included in our ligand-receptor database through continuous updating. Second, it is known that cross species reactivity varies depending on each ligand-receptor interaction $[46,47]$, which potentially leads to false findings. Detailed information regarding such cross-species interactions is needed in the future. Also, human xenograft models usually involve the use of immunodeficient mice, which have greatly reduced number of lymphocytes. Therefore, the interactions between cancer cell and such lymphocytes, which are possibly druggable, will not be covered by the CASTIN analysis.

Despite the above limitations, currently there are no comparable bioinformatics methods, that can perform comprehensive and quantitative analysis of cancer-stroma interactome like CASTIN. It is hopefully expected that CASTIN will accelerate researchers' understanding of the whole picture of cancer-stromal interactome quantitatively and visually, and discover critical interactions that are clinically relevant but couldn't be discovered by sample cancer sequencing analysis so far.

\section{Methods}

\section{Cell culture}

PDAC cell lines KLM-1, MiaPaca-2, PANC-1, PK-8, PK-45P and PK-45H were purchased from RIKEN Bio-
Resource Center (Saitama, Japan), PK-1 and PK-9 were purchased from Tohoku University Cell Resource Center (Sendai, Japan), and Capan-1 was purchased from American Type Culture Collection (ATCC, Manassas, USA). KLM-1, Panc-1, PK-1, PK-8, PK-45P, PK-9, PK-45H cells were cultured in RPMI1640 (WAKO Pure Chemical Industries, Osaka, Japan) supplemented with $10 \%$ FBS and $100 \mathrm{mg} / \mathrm{ml}$ penicillin/streptomycin (WAKO Pure Chemical Industries, Osaka, Japan). Capan-1 was cultured in DMEM (WAKO Pure Chemical Industries, Osaka, Japan) supplemented with $20 \% \mathrm{FBS}, 100 \mathrm{mg} / \mathrm{ml}$ penicillin/streptomycin and $200 \mathrm{mmol} / \mathrm{L}$ L-Alanyl-L-Glutamine (WAKO Pure Chemical Industries, Osaka, Japan). MiaPaca-2 was cultured in DMEM supplemented with $10 \%$ FBS and $100 \mathrm{mg} / \mathrm{ml}$ penicillin/streptomycin.

\section{Animal study}

Five to six weeks old BALB/cAJcl-nu/nu female nude mice (CLEA Japan, Tokyo) were used as the host for cancer xenograft model. Briefly, $5 \times 10^{6}$ cells were suspended in $100 \mu \mathrm{l}$ of phosphate buffer saline (PBS (-)), and were injected subcutaneously into the right flank of mice. The animals were sacrificed when the diameter of tumor reached $5 \mathrm{~mm}$. 


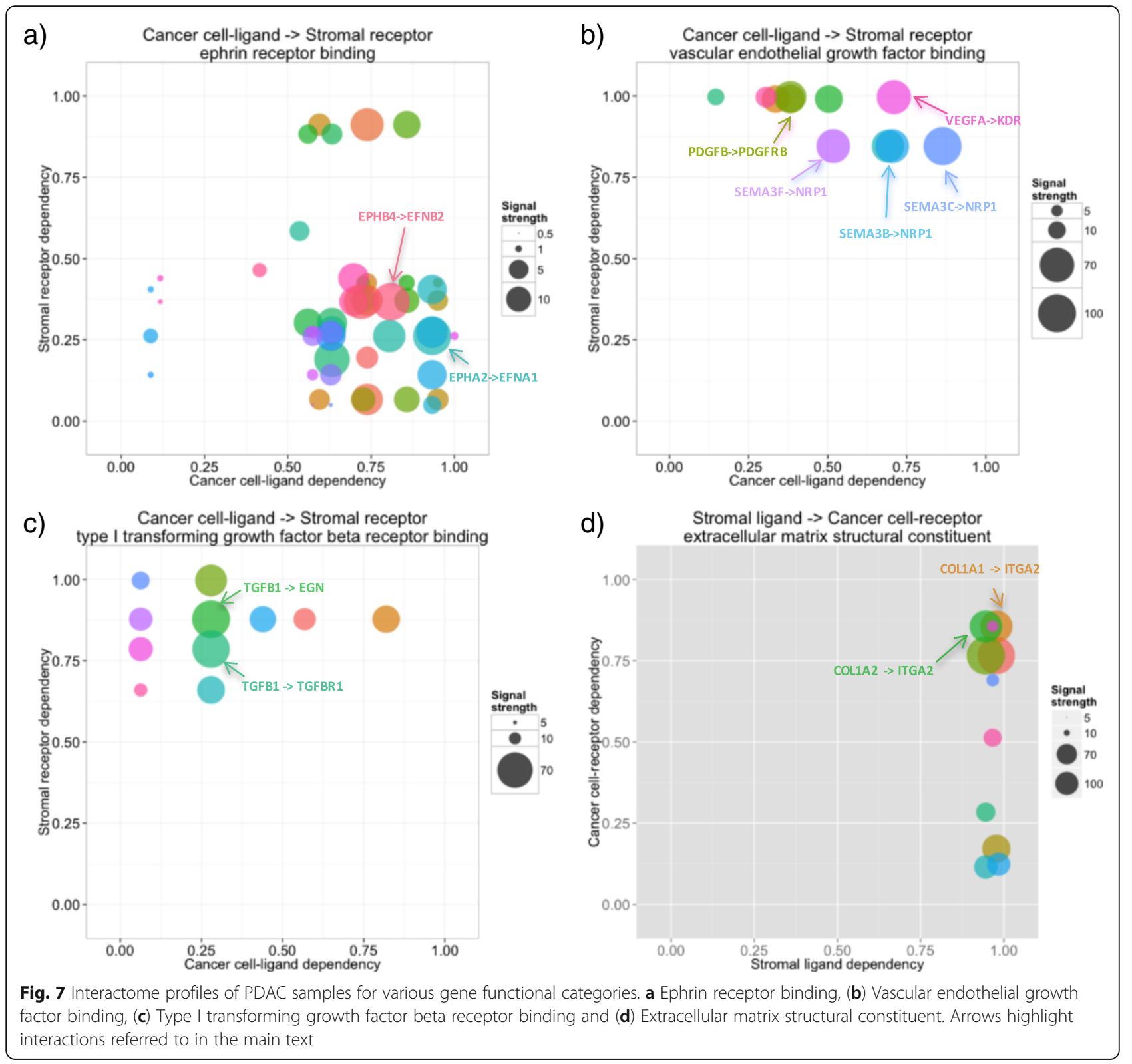

For immunohistochemistry, samples were formalinfixed and embedded in paraffin, and then cut into $7-\mu \mathrm{m}$ thick serial sections and mounted onto microscope slides.

\section{Transcriptome sequencing of xenograft and cell line samples}

Tumors resected from the mice were frozen and the suspended in trizol reagent (Thermo Fisher Scientific Inc, Waltham, USA), and total RNA was extracted according to the manufacturer's instruction. Cultured cells were suspended in trizol reagent (Thermo Fisher Scientific Inc, Waltham, USA), and total RNA was extracted according to the manufacturer's instruction. One microgram of total RNA was used as the starting material for a 50-bp paired-end transcriptome-sequencing protocol using an Illumina GAIIx sequencer (Illumina, San Diego, CA, USA). Briefly, PolyA+ RNA was purified from total RNA and fragmented using divalent cations. RNA quality as assessed by RNA integrity number (RIN) using a bioanalyzer (Agilent), gave a median RIN of 9.0 (ranged from 6.1 to 10). Double stranded cDNA was synthesized using SuperScript II Reverse Transcriptase (Invitrogen), and its overhang was converted into blunt end using T4 DNA polymerase. 3 ' end of the blunt end was adenylated by Klenow fragment, and PE adapter was ligated. Without size selection, the cDNA library was amplified using PCR. For PCR amplification, 1ul of PCR primer PE 1.0 and 2.0 , and $0.5 \mu \mathrm{L}$ of Phusion DNA polymerase (Finnzymes Oy) were used in a final volume of $50 \mu \mathrm{L}$. The 
PCR condition was as follows: $98^{\circ} \mathrm{C}$ for $5 \mathrm{~min}$, then $15 \mathrm{cy}$ cles of $98{ }^{\circ} \mathrm{C}$ for $10 \mathrm{~s}, 65{ }^{\circ} \mathrm{C}$ for $30 \mathrm{~s}$, and $72{ }^{\circ} \mathrm{C}$ for $30 \mathrm{~s}$, followed by $72{ }^{\circ} \mathrm{C}$ for $5 \mathrm{~min}$ before cooling to $4{ }^{\circ} \mathrm{C}$. PCR primers were removed by QIA quick PCR Purification Kit. Each library was loaded into its own single Illumina flow cell lane, producing 50-mer paired-end reads for each sample. The raw sequences have been deposited in the DDBJ Sequence Read Archive under accession number DRA004736.

\section{Transcriptome sequencing of mixture of human and mouse cell lines}

Total RNA of each cell line was extracted and RNA quality was assessed by RIN as described in the previous section. Total RNA from cell lines PANC-1 (human) and SVEC4-10 (mouse) was mixed at the ratios of 1:3, 1:1, and 3:1 ratio. The amount of total RNA was Assayed in the Qubit RNA assay kit (Thermo Fisher Scientific Inc, Waltham, USA).

One microgram of total RNA was used as the starting material for the preparation of transcriptomesequencing library using TruSeq stranded mRNA library preparation kit (Illumina, San Diego, CA, USA) following the manufacturer's directions. Libraries were sequenced 100 bp paired-end on Hiseq2000 sequencer (Illumina). Four libraries were loaded into single lane of Illumina flow cell, producing more than 30 million paired-end reads for each sample. Only the first $50 \mathrm{bp}$ of each paired-end was used for the analysis to compare gene expression levels with the samples sequenced with Illumina GAIIx.

\section{Read mapping and differential taxonomy assignment}

Paired-end reads were aligned to all RefSeq transcripts of human (hg19 coordinates) and mouse (mm10 coordinates) allowing up to one mismatch. Alignments were performed by using TMAP version 3.4.1 [48] with the -a 2 -s 1 -g 3 -u 50 preset. Paired-end reads were considered as RefSeq transcripts if both ends in the pair were mapped to the same RefSeq transcript and each read in the pair was not mapped to other RefSeq transcripts of a different gene. A pair can be mapped to multiple RefSeq transcripts if the condition was met for multiple splice variants of a same gene. NCBI Gene IDs were used to map RefSeq transcripts to genes. Homologene [49] downloaded from NCBI website was used to convert Gene ID of mouse to that of human. When a single human gene was homologous to multiple mouse genes, sum of the expressions of these mouse genes were used.

\section{Quantification of gene expression}

After the read mapping, we removed biases of gene expression levels derived from gene length, distance from poly-A tail, mappability, and regional GC content.
We modeled the count of reads for $j$-th nucleotide of gene $i$ using a Poisson linear model:

$$
\mathrm{E}\left[\log c_{i j}\right]=\log \frac{N_{i} m_{i j}}{\sum_{k} m_{i j}} v_{i}+\alpha g_{i j}+\beta d_{i j}
$$

Where $c_{i j}$ assumed to follow a Poisson distribution is the count of reads covering the $j$-th nucleotide from poly-A tail of gene $i, N_{i}$ is the length of gene $i, m_{i, j}$ is the number of mappable 50 bp covering the $j$-th nucleotide, $v_{i}$ is the true expression of gene $i, v_{i, j}$ is the GC\% around $50 \mathrm{bp}$ of the $j$-th nucleotide, $d_{i, j}$ is the distance from poly-A tail, $\alpha$ is the coefficient of the effect of GC content, and $\beta$ is the coefficient of the effect of distance from poly-A tail. $\alpha$ and $\beta$ depend on experiments, but are independent of genes or nucleotide positions. We assume that all the estimated parameters are identical in human and mouse because sequencing process is the same. $50 \mathrm{bp}$ mappability of each nucleotide was computed using vmatch version 2.0 [50], allowing up to one mismatch. Parameter optimization of the model was performed iteratively as described previously [18]. Initial value of $v_{i}$ was $\sum_{k=1}^{N_{i}^{\prime}} \frac{\sum_{l=1}^{N_{i}^{\prime}} m_{i l}}{N_{i}^{\prime} m_{i k}} c_{i k}$, where $N_{i}^{\prime}=\min \left(N_{i}, 3000\right) . c_{i j}$ is significantly affected by the bias arising from the distance to poly-A tail when $j$ and $N_{i}$ are large, and thus the convergence would be faster if $N_{i}$ instead of $N_{i}$ was used for the initialization. Poisson regression in each iteration was done using a $\mathrm{glm}$ function of $\mathrm{R}$ environment via rJava interface. In order to reduce computational time while maintaining accuracy of the estimated parameters, only transcripts satisfying the following conditions were used for parameter optimization: (i) no splicing variant existed, (ii) the transcript length was more than $8 \mathrm{kbp}$ and (iii) more than $80 \%$ of the transcript was covered with at least 1 read. After parameter optimization, estimated copy number of gene $i$ is calculated as follows:

$$
\frac{{\widetilde{v_{i}}}^{\prime}=\frac{v_{i}}{Z}=\sum_{k=1}^{N_{i}} c_{i k}^{\prime}}{Z \sum_{k=1}^{N_{i}} \exp \left(\alpha g_{i k}+\beta d_{i k}\right)}
$$

where $c_{i j}^{\prime}$ is the count of reads starting at the $j$-th nucleotide of gene $i$ and $Z$ is a normalization factor so that sum of ${\widetilde{v_{i}}}^{\prime}$ below the 95th percentile be 300,000, which is roughly the average number of mRNA molecules present in a cell [51]. Note that $c_{i j}$ instead of $c_{i j}$ was used in the estimation step because the effect of $\mathrm{GC} \%$ was expected to be corrected more accurately. Conversely, $c_{i j}$ was used in the optimization step since $c_{i j}$ was so sparse that the parameter could not be estimated accurately. 


\section{Indices for interactome data evaluation}

For the purpose of quantitative and comprehensive evaluation of interactome, we have introduced three evaluation indices for each signal direction for each gene. We assume that there are $P$ pairs of ligand and receptor genes in our in-house database. Let $L_{C i}, L_{S i}, R_{C j}$, and $R_{S j}$ be normalized gene expression levels of ligand gene $i(i=1, \ldots, P)$ of human (cancer), ligand gene $i(i=1, \ldots, P)$ of mouse (stroma), receptor gene $j(j=1, \ldots, P)$ of human (cancer), receptor gene $j(j=1, \ldots, P)$ of mouse (stroma), respectively. We define three evaluation indices, ligand dependency $X$, receptor dependency $Y$, signal strength $Z$ for each direction as follows:

- C-S direction

$$
\begin{aligned}
\mathrm{X}_{\mathrm{C} \rightarrow \mathrm{S}, i} & =\frac{L_{C i}}{L_{C i}+L_{S i}} \\
\mathrm{Y}_{\mathrm{C} \rightarrow \mathrm{S}, j} & =\frac{R_{S j}}{R_{C j}+R_{S j}} \\
\mathrm{Z}_{C \rightarrow S, i, j} & =\sqrt{L_{C i} \cdot R_{S j}}
\end{aligned}
$$

- S-C direction

$$
\begin{aligned}
& \mathrm{X}_{S \rightarrow C, i}=\frac{L_{S i}}{L_{C i}+L_{S i}} \\
& \mathrm{Y}_{S \rightarrow C, j}=\frac{R_{C j}}{R_{C j}+R_{S j}} \\
& \mathrm{Z}_{S \rightarrow C, i, j}=\sqrt{L_{S i}} \cdot R_{C j}
\end{aligned}
$$

\section{In-house ligand-receptor database construction}

We have constructed an in-house ligand-receptor database. The database construction consisted of three main steps (i) extraction of localization information from Human Protein Reference Database (HPRD) [20] (ii) extraction of ligand-receptor interaction from Kyoto Encyclopedia of Genes and Genomes (KEGG) data [19] (iii) curation by reviewing original literature.

First, proteins localized primarily to extracellular space and plasma membrane were selected as ligand and receptor candidates, respectively. Information of primary localization was downloaded from Human Protein Reference Database (HPRD, release 8) [20] on 9 September 2009.

Among all the pairs of ligand and receptor candidates, only those appeared in protein-protein interaction in Kyoto Encyclopedia of Genes and Genomes (KEGG) pathway database [19] (release 55.0, downloaded on 7 August 2010) proceeded to the next curation step.
Direction of interaction was determined according to relations (activation, inhibition, binding/association, or indirect effect) in KEGG database. For example, if 'A activates B' appeared, A and B became candidates of ligand and receptor, respectively. If the relationship was undirectional such as 'binding/association', direction was determined at random with one exception: proteins in Ephrin and Ephrin-receptor families appeared in Axon guidance pathway (Entry: hsa04360), were assigned as ligand and receptor, and vice versa [36]. Interactions occurring within the same cell were removed manually.

Finally, researchers in the field of biology curated each interaction by carefully reviewing the original literature attached in the KEGG database.

\section{Visualization interface of the CASTIN output}

We have also developed a web interface for interactive 2D-visualization of the CASTIN output (http://gpatgaze za.tmd.ac.jp/CASTIN_viewer/index.php). Here we introduce a brief description of the interface (Additional file 3: Figure S2). Please refer to the manual for detail.

There are two 2D scatter plots, each of which corresponding to $\mathrm{S}-\mathrm{C}$ and $\mathrm{C}-\mathrm{S}$ direction of signals. In each scatter plot, horizontal axis represents ligand dependency and vertical axis represents receptor dependency. Each circle represents ligand-receptor interactions, with its radius proportional to the log of signal strength of the interaction.

When users hover cursor over the circles, gene symbols of the ligand and the receptor and its signal strength is shown.

By inputting the threshold value of signal strength in "Threshold of signal strength" box and pressing "View" button, users can hide weak (and thus possibly nonsignificant) interactions. Additionally, users can search specific genes by entering gene symbol (s) in "Search Genes (Gene Symbol)" box.

\section{Gene ontology analysis}

We used all the gene ontology categories of human, irrespective of their hierarchies. All gene ontology categories and the genes belonging to each category were retrieved from Gene Ontology Consortium [52] on February 10, 2016.

\section{Immunohistochemistry}

Cut specimens of formalin-fixed and paraffin-embedded mouse tumors (Capan-1, KLM-1, MiaPaCa-2, PANC-1, PK-1, PK-8, PK-9 and PK45-P) were obtained from tumor transplanted mice as described above. After de-paraffinized by Xylene (Wako Pure Chemical Industries, Japan) for $10 \mathrm{~min}$ at room temperature, the specimen slides were treated with Citrate buffer $(\mathrm{pH}$ 6.0) (Abcam, 
UK) by an autoclave (TOMY Seiko, Japan) at $121{ }^{\circ} \mathrm{C}$ for $5 \mathrm{~min}$ in order to retrieve protein antigens. Endogenous peroxidase activity was masked by incubating the slides with $3 \% \mathrm{H}_{2} \mathrm{O}_{2}$ (Sigma Aldrich, USA) for $10 \mathrm{~min}$ at room temperature. The slides were incubated with $2 \%$ BSA (Sigma Aldrich) / PBS for $1 \mathrm{~h}$ at room temperature to block non-specific protein-antibody reactions. Then the slides were incubated with anti-FABP5 antibody (Rabbit \#39926, Cell Signaling Technology, USA) at $1 / 200$ dilution for an over-night at $4{ }^{\circ} \mathrm{C}$. Histostar (MBL, Japan) and DAB solution (MBL) were used to detect the FABP5 $1^{\text {st }}$ antibody signals under a microscope (Olympus, Japan) with the nuclear staining with Hematoxylin (Sakura Finetek Japan, Japan).

\section{Statistical tests}

All the $p$-values were calculated by Binomial test (onesided) and transformed into $q$-values for false discovery rate (FDR) analysis using the 'qvalue' package from Bioconductor.

\section{Endnotes}

${ }^{1}$ Hepatocyte growth factor

${ }^{2}$ MET proto-oncogene, receptor typrosine kinase

${ }^{3}$ Epidermal growth factor

${ }^{4}$ Epidermal growth factor receptor

${ }^{5}$ Vascular endothelial growth factor A

${ }^{6}$ Kinase insert domain receptor

${ }^{7}$ Vascular endothelial growth factor B

${ }^{8} \mathrm{FMS}$ related tyrosine kinase 1

\section{Additional files}

Additional file 1: Figure S1. Read count biases in a xenograft sample from PDAC cell line (PK-1). Randomly chosen 100,000 read count residuals from fitted model are plotted against $(a, b)$ distance from poly-A site and $(c, d)$ regional GC content. Residuals with biases $(a, c)$ and without biases (b, d). Randomly chosen 100,000 points are plotted. Straight line in each plot indicates the bias estimated from 200 genes using Poisson linear model (see Methods). (PDF $70 \mathrm{~kb}$ )

Additional file 2: Table S1. Ligand-receptor interactions used in the CASTIN system. (PDF $175 \mathrm{~kb}$ )

Additional file 3: Figure S2. Screenshot of the CASTIN interactive viewer. Hover a cursor over a circle and it displays the interacting genes represented and their value of signal strength. (PDF $1376 \mathrm{~kb}$ )

Additional file 4: Table S2. Summary statistics of RNA-seq for mixture of total RNA from human (PANC-1) and mouse (SVEC4-10) cell lines. (PDF $37 \mathrm{~kb}$ )

Additional file 5: Figure S3. Comparison of gene expression levels of samples with different RNA mixture ratios (PANC-1 and SVEC4-10) estimated by CASTIN. (a) gene expression levels of human (human content: $25 \%, 50 \%, 75 \%, 100 \%$ ) and (b) mouse (mouse content: $100 \%$, $75 \%, 50 \%, 25 \%)$ after global normalization in each species. On the bottom of the diagonal: the bivariate scatter plots with the identity line. On the top of the diagonal: the value of the cosine correlation. (PDF $608 \mathrm{~kb}$ )

Additional file 6: Figure S4. FABP5/Fabp5 expression in RNA-Seq (estimated by CASTIN) and Immunohistochemistry. (a) gene expression levels. (b) Immunohistochemical (IHC) and hematoxylin and eosin (H\&E) staining of close sections. Sections of PDAC xenograft cancer derived from each cell line were stained for FABP5/Fabp5 (left) or with H\&E (right). The slightly different distribution of tumor and stromal cells between H\&E and the corresponding $\mathrm{HC}$ sections was due to the physical distance between the two sections. (PDF $10295 \mathrm{~kb}$ )

Additional file 7: Figure S5. Distribution of hedgehog-related interactions in PDAC samples. a) Cancer cell SHH to stromal PTCH1. b) Cancer-cell IHH to stromal PTCH1. c) Cancer-cell IHH to stromal PTCH2. (PDF $162 \mathrm{~kb}$ )

Additional file 8: Figure S6. Distribution of mutually dependent interactions with strong signals in PDAC samples. a) Stromal SEMA3C to cancer-cell PLXNB1. b) Cancer-cell SEMA4D to stromal NRP1. (PDF $136 \mathrm{~kb}$ )

\section{Abbreviations}

FDR: False discovery rate; GO: Gene ontology; GSEA: Gene set enrichment analysis; HPRD: Human protein reference database; KEGG: Kyoto encyclopedia of genes and genomes; PBS: Phosphate buffer saline; PDAC: Pancreas ductal adenocarcinoma; RIN: RNA integrity number; TPM: Transcripts per kilobase million

\section{Acknowledgments}

We thank Kaori Shiina, Kaoru Nakano, Kei Sakuma, and Ken Tominaga for their technical assistance.

\section{Funding}

This study was supported by JSPS Grants-in-Aid for Young Scientists (A), No. 16H02481 and Grant-in-Aid for Scientific Research (A), No. 25710020 and (B), No 15H04287, by AMED Practical Research for Innovative Cancer Control, No. 4 K112 (SI), and by the Joint Usage/Research Program of Medical Research Institute, Tokyo Medical and Dental University (DK, SI).

\section{Availability of data and materials}

The RNA-Seq data supporting the results of this article have been deposited in the Sequence Read Archive of DDBJ under the accession number DRA004736.

The CASTIN software is implemented in Java 7 and Ruby 2.0. It depends on R and rJava package for Poisson regression. The user also needs a short-reader alignment software (e.g., bowtie) for preparing input files, and a pairwise alignment software (e.g., vmatch) for pre-calculating mappability of reference sequence. The CASTIN software and the source code are freely available at http://github.com/tmd-gpat/CASTIN (DOI: 10.5281/zenodo.51156) under the GNU General Public License (GPL).

\section{Authors' contributions}

SI conceived and designed the experiments. DK and RSuzuki developed the CASTIN software. KK developed the visualization system of CASTIN. SI, TI, and RSato performed the experiments. SY, KT, and HA contributed to the data generation. DK analyzed the data. HK performed histological analysis. SI and MT contributed to the manual curation of the database. DK and TI wrote the paper. SI, MF, and HA critically read the manuscript and discussed the results. All of the authors have read and approved the final manuscript.

\section{Competing interests}

The authors declare that they have no competing interests.

\section{Consent for publication}

Not applicable.

\section{Ethics approval and consent to participate}

All animal experiments were approved by Tokyo Medical and Dental University Ethics Committee for Animal Experiments and strictly adhered to the animal experiment guidelines of Tokyo Medical and Dental University.

\section{Author details}

'Department of Genomic Pathology, Medical Research Institute, Tokyo Medical and Dental University, Tokyo, Japan. ${ }^{2}$ Graduate School of Interdisciplinary Information Studies, The University of Tokyo, Tokyo, Japan. ${ }^{3}$ Graduate School of Information and Science and Technology, The University of Tokyo, Tokyo, Japan. ${ }^{4}$ Department of Pathology, Graduate School of 
Medicine, The University of Tokyo, Tokyo, Japan. ${ }^{5}$ Genome Science Division, Research Center for Advanced Science and Technology, The University of Tokyo, Tokyo, Japan.

\section{Received: 9 June 2016 Accepted: 25 October 2016} Published online: 09 November 2016

\section{References}

1. Quail D, Joyce J. Microenvironmental regulation of tumor progression and metastasis. Nat Med. 2013;19:1423-37

2. Tchou J, Conejo-Garcia J. Targeting the tumor stroma as a novel treatment strategy for breast cancer: shifting from the neoplastic cell-centric to a stroma-centric paradigm. Adv Pharmacol San Diego Calif. 2012;65:45-61.

3. Li X, Ma Q, Xu Q, Duan W, Lei J, Wu E. Targeting the cancer-stroma interaction: a potential approach for pancreatic cancer treatment. Cur Pharm Des. 2012;18:2404-15.

4. McMillin DW, Negri JM, Mitsiades CS. The role of tumour-stromal interactions in modifying drug response: challenges and opportunities. Nat Rev Drug Discov. 2013;12:217-28.

5. Olive KP, Jacobetz MA, Davidson CJ, Gopinathan A, Mclntyre D, Honess D, et al. Inhibition of hedgehog signaling enhances delivery of chemotherapy in a mouse model of pancreatic cancer. Science. 2009:324:1457-61.

6. Kandoth C, McLellan MD, Vandin F, Ye K, Niu B, Lu C, et al. Mutational landscape and significance across 12 major cancer types. Nature. 2013;502 333-9.

7. Bradford JR, Farren M, Powell SJ, Runswick S, Weston SL, Brown H, et al. RNA-Seq differentiates tumour and host mRNA expression changes induced by treatment of human tumour xenografts with the VEGFR tyrosine kinase inhibitor cediranib. PLoS One. 2013:8:e66003.

8. Siolas D, Hannon GJ. Patient-derived tumor xenografts: transforming clinical samples into mouse models. Cancer Res. 2013:73:5315-9.

9. Makałowski W, Zhang J, Boguski MS. Comparative analysis of 1196 orthologous mouse and human full-length mRNA and protein sequences. Genome Res. 1996;6:846-57.

10. Thijssen VLJL, Brandwijk RJMGE, Dings RPM, Griffioen AW. Angiogenesis gene expression profiling in xenograft models to study cellular interactions. Exp Cell Res. 2004:299:286-93.

11. Boedigheimer MJ, Freeman DJ, Kiaei P, Damore MA, Radinsky R. Gene expression profiles Can predict panitumumab monotherapy responsiveness in human tumor xenograft models. Neoplasia N Y N. 2013;15:125-32.

12. Hollingshead MG, Stockwin LH, Alcoser SY, Newton DL, Orsburn BC, Bonomi CA, et al. Gene expression profiling of 49 human tumor xenografts from in vitro culture through multiple in vivo passages - strategies for data mining in support of therapeutic studies. BMC Genomics. 2014:15:393.

13. Rajaram M, Li J, Egeblad M, Powers RS. System-wide analysis reveals a complex network of tumor-fibroblast interactions involved in tumorigenicity. PLoS Genet. 2013;9:e1003789.

14. Creighton CJ, Bromberg-White JL, Misek DE, Monsma DJ, Brichory F, Kuick R, et al. Analysis of tumor-host interactions by gene expression profiling of lung adenocarcinoma xenografts identifies genes involved in tumor formation. Mol Cancer Res MCR. 2005:3:119-29.

15. Henare K, Wang L, Wang L-C, Thomsen L, Tijono S, Chen C-J, et al. Dissection of stromal and cancer cell-derived signals in melanoma xenografts before and after treatment with DMXAA. Br J Cancer. 2012;106: 1134-47.

16. Li B, Dewey CN. RSEM: accurate transcript quantification from RNA-Seq data with or without a reference genome. BMC Bioinformatics. 2011;12:323.

17. Risso D, Schwartz K, Sherlock G, Dudoit S. GC-content normalization for RNA-Seq data. BMC Bioinformatics. 2011;12:480.

18. Li J, Jiang $H$, Wong WH. Modeling non-uniformity in short-read rates in RNA-Seq data. Genome Biol. 2010;11:R50.

19. Kanehisa M, Goto S. KEGG: kyoto encyclopedia of genes and genomes. Nucleic Acids Res. 2000;28:27-30

20. Keshava Prasad TS, Goel R, Kandasamy K, Keerthikumar S, Kumar S, Mathivanan S, et al. Human protein reference database-2009 update. Nucleic Acids Res. 2009;37:D767-72

21. Whatcott CJ, Diep CH, Jiang P, Watanabe A, LoBello J, Sima C, et al. Desmoplasia in primary tumors and metastatic lesions of pancreatic cancer. Clin Cancer Res. 2015;21:3561-8. clincanres.1051.2014.

22. Huang M, Shen A, Ding J, Geng M. Molecularly targeted cancer therapy: some lessons from the past decade. Trends Pharmacol Sci. 2014;35:41-50.
23. Muckenhuber A, Babitzki G, Thomas M, Hölzlwimmer G, Zajac M, Jesinghaus $M$, et al. Profiling of cMET and HER Family Receptor Expression in Pancreatic Ductal Adenocarcinomas and Corresponding Lymph Node Metastasis to Assess Relevant Pathways for Targeted Therapies: Looking at the Soil Before Planting the Seed. Pancreas. 2016;45:1167-74.

24. Park SJ, Gu MJ, Lee DS, Yun SS, Kim HJ, Choi JH. EGFR expression in pancreatic intraepithelial neoplasia and ductal adenocarcinoma. Int J Clin Exp Pathol. 2015;8:8298-304.

25. Brown LF, Guidi AJ, Tognazzi K, Dvorak HF. Vascular permeability factor/ vascular endothelial growth factor and vascular stroma formation in neoplasia. Insights from in situ hybridization studies. J Histochem Cytochem. 1998;46:569-75

26. Kim EJ, Sahai V, Abel EV, Griffith KA, Greenson JK, Takebe N, et al. Pilot clinical trial of hedgehog pathway inhibitor GDC-0449 (vismodegib) in combination with gemcitabine in patients with metastatic pancreatic adenocarcinoma. Clin Cancer Res. 2014;20:5937-45.

27. Schwarz Q, Ruhrberg C. Neuropilin, you gotta let me know: should I stay or should I go? Cell Adhes Migr. 2010;4:61-6.

28. Ellis LM. The role of neuropilins in cancer. Mol Cancer Ther. 2006:5:1099-107.

29. Banu N, Teichman J, Dunlap-Brown M, Villegas G, Tufro A. Semaphorin $3 C$ regulates endothelial cell function by increasing integrin activity. FASEB J. 2006;20:2150-2.

30. Yaqoob U, Cao S, Shergill U, Jagavelu K, Geng Z, Yin M, et al. Neuropilin-1 stimulates tumor growth by increasing fibronectin fibril assembly in the tumor microenvironment. Cancer Res. 2012;72:4047-59.

31. Järveläinen $H$, Sainio $A$, Koulu $M$, Wight TN, Penttinen R. Extracellular matrix molecules: potential targets in pharmacotherapy. Pharmacol Rev. 2009;61: 198-223.

32. Armstrong T, Packham G, Murphy LB, Bateman AC, Conti JA, Fine DR, et al. Type I collagen promotes the malignant phenotype of pancreatic ductal adenocarcinoma. Clin Cancer Res. 2004;10:7427-37.

33. Giordano S, Corso S, Conrotto P, Artigiani S, Gilestro G, Barberis D, et al. The semaphorin $4 \mathrm{D}$ receptor controls invasive growth by coupling with Met. Nat Cell Biol. 2002;4:720-4.

34. Kato S, Kubota K, Shimamura T, Shinohara Y, Kobayashi N, Watanabe S, et al Semaphorin 4D, a lymphocyte semaphorin, enhances tumor cell motility through binding its receptor, plexinB1, in pancreatic cancer. Cancer Sci. 2011;102:2029-37.

35. Capparuccia L, Tamagnone L. Semaphorin signaling in cancer cells and in cells of the tumor microenvironment - two sides of a coin. J Cell Sci. 2009:122:1723-36.

36. Pasquale EB. Eph receptors and ephrins in cancer: bidirectional signaling and beyond. Nat Rev Cancer. 2010;10:165-80.

37. Korc M. Pathways for aberrant angiogenesis in pancreatic cancer. Mol Cancer. 2003;2:8.

38. Yuzawa S, Kano MR, Einama T, Nishihara H. PDGFRß expression in tumor stroma of pancreatic adenocarcinoma as a reliable prognostic marker. Med Oncol. 2012:29:2824-30

39. Seo Y, Baba H, Fukuda T, Takashima M, Sugimachi K. High expression of vascular endothelial growth factor is associated with liver metastasis and a poor prognosis for patients with ductal pancreatic adenocarcinoma. Cancer. 2000;88:2239-45.

40. Korc M. Pancreatic cancer associated stroma production. Am J Surg. 2007; 194:s84-6

41. Kojima Y, Acar A, Eaton EN, Mellody KT, Scheel C, Ben-Porath I, et al. Autocrine TGF- $\beta$ and stromal cell-derived factor-1 (SDF-1) signaling drives the evolution of tumor-promoting mammary stromal myofibroblasts. Proc Natl Acad Sci. 2010;107:20009-14.

42. Löhr M, Schmidt C, Ringel J, Kluth M, Müller P, Nizze H, et al. Transforming growth factor-beta1 induces desmoplasia in an experimental model of human pancreatic carcinoma. Cancer Res. 2001;61:550-5.

43. Medicherla S, Li L, Ma JY, Kapoun AM, Gaspar NJ, Liu Y-W, et al. Antitumor activity of TGF-beta inhibitor is dependent on the microenvironment. Anticancer Res. 2007:27:4149-57.

44. Fonsatti E, Sigalotti L, Arslan P, Altomonte M, Maio M. Emerging role of endoglin (CD105) as a marker of angiogenesis with clinical potential in human malignancies. Curr Cancer Drug Targets. 2003;3:427-32.

45. Yoshitomi H, Kobayashi S, Ohtsuka M, Kimura F, Shimizu H, Yoshidome H, et al. Specific expression of endoglin (CD105) in endothelial cells of intratumoral blood and lymphatic vessels in pancreatic cancer. Pancreas. 2008:37:275-81.

46. Layton MJ, Lock P, Metcalf D, Nicola NA. Cross-species receptor binding characteristics of human and mouse leukemia inhibitory factor suggest a complex binding interaction. J Biol Chem. 1994;269:17048-55. 
47. Herren B, Weyer KA, Rouge M, Lötscher P, Pech M. Conservation in sequence and affinity of human and rodent PDGF ligands and receptors. Biochim Biophys Acta. 1993;1173:294-302.

48. iontorrent/TMAP [Internet]. GitHub. [cited 2015 Apr 15]. Available from: https://github.com/iontorrent/TMAP

49. Resource NCBI. Coordinators. Database resources of the National center for biotechnology information. Nucleic Acids Res. 2016:44:D7-D19.

50. Kurtz S. The Vmatch large scale sequence analysis software [Internet]. [cited 2016 May 16]. Available from: http://www.vmatch.de/

51. Velculescu VE, Madden SL, Zhang L, Lash AE, Yu J, Rago C, et al. Analysis of human transcriptomes. Nat Genet. 1999;23:387-8.

52. Gene Ontology Consortium. Gene ontology consortium: going forward. Nucleic Acids Res. 2015;43:D1049-56.

53. Capurro MI, Xiang Y-Y, Lobe C, Filmus J. Glypican-3 promotes the growth of hepatocellular carcinoma by stimulating canonical Wnt signaling. Cancer Res. 2005;65:6245-54.

54. Jiang W, Zhang Y, Kane KT, Collins MA, Simeone DM, di Magliano MP, et al. CD44 regulates pancreatic cancer invasion through MT1-MMP. Mol Cancer Res MCR. 2015;13:9-15.

55. Li W, Liu Z, Zhao C, Zhai L. Binding of MMP-9-degraded fibronectin to $\beta 6$ integrin promotes invasion via the FAK-Src-related Erk1/2 and PI3K/Akt/ Smad-1/5/8 pathways in breast cancer. Oncol Rep. 2015;34:1345-52.

56. Kim B-K, Yoo H-I, Kim I, Park J, Kim YS. FZD6 expression is negatively regulated by miR-199a-5p in human colorectal cancer. BMB Rep. 2015;48: 360-6.

57. Subramani R, Lopez-Valdez R, Arumugam A, Nandy S, Boopalan T, Lakshmanaswamy $R$. Targeting insulin-like growth factor 1 receptor inhibits pancreatic cancer growth and metastasis. PLoS One. 2014;9:e97016.

\section{Submit your next manuscript to BioMed Central} and we will help you at every step:

- We accept pre-submission inquiries

- Our selector tool helps you to find the most relevant journal

- We provide round the clock customer support

- Convenient online submission

- Thorough peer review

- Inclusion in PubMed and all major indexing services

- Maximum visibility for your research

Submit your manuscript at www.biomedcentral.com/submit 